\title{
Rosettes within rosacea
}

\section{Niema Aqil, Aicha Nassiri, Kaoutar Moustaide, Salim Gallouj, Fatima Zahra Mernissi}

\author{
Department of dermatology, Hassan II University Hospital Center, Fes, Morocco
}

Corresponding author: Dr. Niema Aqil, E-mail: niemaaqil90@gmail.com

Sir,

A 43-year-old woman presented with recurrent flushing of the face and redness, erythematous lesions of the face with hypersensitivity to heat. These symptoms had persisted for 4 years, with intermittent remissions lasting up to 2 months. A physical examination revealed facial erythema, telangiectasia, papules and pustules of the midfacial region with some scales and crusts. (Fig. 1) Dermoscopy revealed linear vessels characteristically arranged in a polygonal network, creamy and whitish linear areas and a clear rosette sign. (Fig. 2) The rest of the somatic examination was without abnormalities. An ophthalmological examination showed no evidence of keratitis, conjunctivitis or blepharitis. On these bases a diagnosis of papulopustular rosacea was made. The patient was treated with doxycycline for a total of 12 weeks, which led to a significant improvement.

The rosette sign has been previously observed by Cuellar and colleagues and has been described as a new dermoscopic sign in actinic keratoses, which may be due to alternating areas of orthokeratosis and parakeratosis [1]. Recently, Liebman and his collaborators have pointed out that the rosette sign is an optical effect of polarized light and that its interaction with keratin-filled adnexal openings is observable in a wide range of cutaneous neoplasia [2]. This correlation could also explain the presence of this sign in rosacea too.

\section{Consent}

The examination of the patient was conducted according to the Declaration of Helsinki principles.

\section{REFERENCES}

1. Cuellar F, Vilalta A, Puig S, Palou J, Salerni G, Malvehy J. New dermoscopic pattern in actinic keratosis and related conditions. Arch Dermatol. 2009; 145: 732.

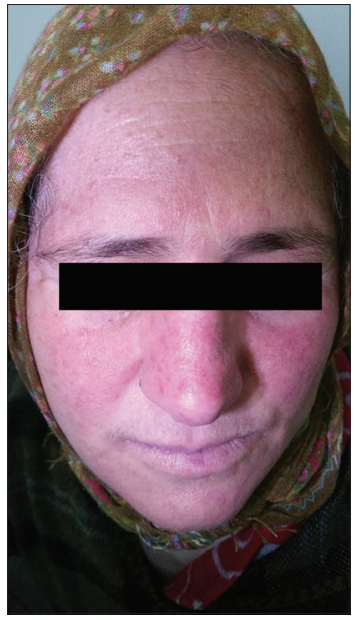

Figure 1: Facial erythema, telangiectasia, papules and pustules of the midfacial region with some scales and crusts.

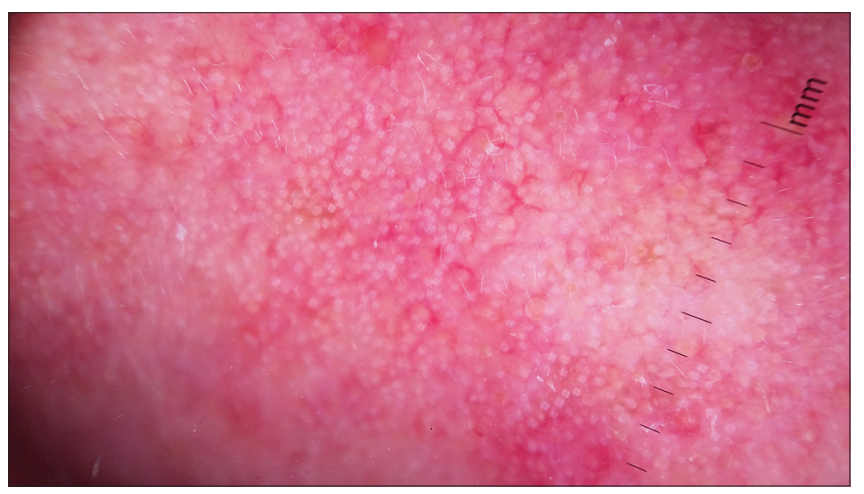

Figure 2: Dermoscopy showing linear vessels characteristically arranged in a polygonal network, creamy and whitish linear areas and a clear rosette sign.

2. Liebman TN, Scope A, Rabinovitz H, Braun RP, Marghoob AA. Rosettes may be observed in a range of conditions. Arch Dermatol. 2011;147:1468.

Copyright by Niema Aqil, et al. This is an open-access article distributed under the terms of the Creative Commons Attribution License, which permits unrestricted use, distribution, and reproduction in any medium, provided the original author and source are credited.

Source of Support: Nil, Conflict of Interest: None declared. 